\title{
X-Ray photoelectron spectroscopic investigation of phenosafranine adsorbed onto micro and mesoporous materials ${ }^{\dagger}$
}

\author{
S EASWARAMOORTHI ${ }^{1}$, K ANANTHANARAYANAN $^{1}$, B SREEDHAR $^{2}$ and \\ P NATARAJAN ${ }^{1} *$ \\ ${ }^{1}$ National Centre for Ultrafast Processes, University of Madras, Taramani Campus, Chennai 600113 \\ ${ }^{2}$ Indian Institute of Chemical Technology, Uppal Road, Hyderabad 500607 \\ e-mail: pnatrajan $a$ hotmail.com
}

\begin{abstract}
The phenosafranine adsorbed onto the micro and mesoporous materials prepared by ion exchange method and interaction of the dye with host materials were studied by X-ray photoelectron spectroscopy to elucidate the influence of the host matrix on the binding energy of $\mathrm{N} 1 \mathrm{~s}$ orbital. Core level $\mathrm{N}$ 1s X-ray photoelectron spectroscopy reveals the interaction between the dye and the solid surface through the hydrogen bonding between the hydrogen atoms of primary amino groups in dye molecule and the oxygen atom of surface hydroxyl groups. The strength of the hydrogen bonding depends on the nature of the solid surface. In the dye adsorbed onto the micro and mesoporous materials the interaction between adsorbed phenosafranine and the surfaces of the porous materials are found to modify the optical spectra and the excited state dynamics of the confined phenosafranine molecules. The change in photophysical properties of phenosafranine adsorbed on to the host materials on dehydration at elevated temperatures is attributed to the modification of host surface during dehydration process.
\end{abstract}

Keywords. Phenosafranine; X-ray photoelectron spectroscopy; mesoporous materials.

\section{Introduction}

Research involving micro and mesoporous materials as host material is of great interest with potential applications in catalysis, adsorbents, chemical sensors, optical/electronic nanodevices and other areas. ${ }^{1-3}$ Mesoporous materials enable the encapsulation of metal complexes and organic dye molecules in the channels and cavities of nanodimension in these materials. Organisation of molecular species into highly ordered architectures has attracted increasing attention from a wide range of scientific aspects and practical applications. ${ }^{4}$ The construction of the functional materials by organising photoactive species with highly ordered structure has actively been investigated for light induced reactions. ${ }^{5}$ The arrangement and spatial location of the encapsulated photoactive species are influenced by the structure of the host material. The photophysical and photochemical reactions in such heterogeneous system have been observed to be significantly different from the analogous reactions in homogeneous solutions. The nanoscopic structure of the assembly of the photoactive species

\footnotetext{
${ }^{\dagger}$ Dedicated to the memory of the late Professor S K Rangarajan *For correspondence
}

could be tailored by selecting and designing both the guests and hosts and also by co-adsorption processes.

Among the various micro heterogeneous systems studied, molecular sieves are ideal host for supramolecular organisation by providing high chemical, mechanical and thermal stability. The defined geometry of the cavities and channels are essential for a specific spatial arrangement of guest species, thereby generating highly structured composite materials. In order to develop such photochemical systems, an understanding of the host-guest interaction between the molecules and the molecular sieves is important. The influence of various parameters such as confinement effect of these sieves on the guest molecule, interactions with cationic sites and the framework becomes significant. ${ }^{6}$ The photophysical and photochemical behaviour of organic dyes encapsulated in micro and mesoporous materials are widely studied. ${ }^{7,8}$ The structural characteristics of these designed materials enable the incorporation of optically active guest molecules in crystallographically defined positions and highly organised arrangements. ${ }^{9}$ MCM-41 is a versatile host owing to its high thermal stability, high surface area, high pore volume and very narrow pore size distribution tunable in the range of $20-100 \AA$. A structural model for 
MCM-41 consists of a hexagonal arrangement of cylindrical pores embedded in a matrix of amorphous silica.

Electronic absorption and emission spectral studies of molecular-sieve-encapsulated chromophores have been of immense value to understand the influence of the environment on the electronic states of the guest molecules. X-ray photoelectron spectroscopy (XPS) is an important tool to understand the interaction of the guest species with solid host materials. Encapsulation of organic molecules in molecular sieves leads to changes in their optical, physical and chemical properties. ${ }^{10}$ The photophysical and photochemical behaviour of organic dyes encapsulated in mesoporous materials have been widely studied. Spectroscopic studies of rhodamine B doped composites have been reported by Hoppe et $a l^{11}$. Yamashita et $a l$ have observed that the lifetime of rhodomine B in MCM-41 was longer than that observed on silica gel or in ZSM-5 hosts. ${ }^{12}$ Photoluminescence properties of coumarin 540 doped in MCM-41 was studied by Gu et al, ${ }^{13}$ which compared the photophysical properties of the dye in MCM-41 with those in ethanol solution. Photophysical studies on tris(2,2'-bipyridine) ruthenium(II) complex loaded in mesoporous silica were carried out by Ogawa et $a l .{ }^{14} \mathrm{~A}$ rare earth complex recently encapsulated in mesoporous silicates showed that the photophysical properties are sensitive to the local environment. ${ }^{15}$ Aggregation of the thionine dye within AlMCM-48 was reported earlier. ${ }^{16}$ Investigation on the spectral properties and photophysics of a laser dye 4-(dicyanomethylene)-2-methyl-6-( $p$-dimethylaminostyryl)-4H-pyran encapsulated in MCM-41 mesoporous material and Zeolite-Y has been reported. ${ }^{17}$ Photophysical properties of some coumarin derivatives incorporated in MCM-41 were studied by Li et al recently. ${ }^{18}$ The energy transfer process between the dye molecules in the nanochannels of MCM-41 is currently gathering momentum. Anpo et al recently investgated the energy transfer between two organic dyes occluded in the nanochannels of different mesoporous hosts. ${ }^{19}$ A dumbbell-shaped multicomponent system consisting of a ruthenium(II) polypyridine complex, a p-terphenyl, a 4,4'-bipyridinium unit, a 3,3'-dimethyl-4,4'-bipyridinium unit, and a tetraarylmethane unit has been enclosed in the channels of an AlMCM-41 aluminosilicate. $^{20}$ Electron transfer from the excited ruthenium(II) complex to the substrate in silicate matrix was observed. Kispert et al studied the electron transfer reactions of carotenoids, $\beta$-carotene, canthaxanthin and 7'-apo-7', 7'dicyano- $\beta$-carotene embedded in MCM-41 and TiMCM-41. ${ }^{21}$

We report in this paper the preparation and characterization of phenosafranine dye adsorbed onto micro and mesoporous materials. The nature of interaction between the dye and the host materials has been investigated by $\mathrm{X}$-ray photoelectron spectroscopy. Phenosafranine belongs to azine family of dyes, which acts as an electron transfer reagent in the excited state. ${ }^{22}$ Photosensitisation process of this dye has been reported for application in photogalvanic cells ${ }^{23}$ and the sensitisation of bulk titanium dioxide nanoparticles. ${ }^{24}$ The environment sensitive fluorescent property of phenosafranine was used to probe the reverse micellar system ${ }^{25}$ and also the covalently attached dye was used to study the dynamics of poly(carboxylic acids) in dilute aqueous solutions. ${ }^{26}$

\section{Experimental methods}

\subsection{Chemicals}

Phenosafranine $\left(\mathrm{PS}^{+}\right)$chloride 1 (3,7-diamino-5phenylphenazenium chloride) obtained from Aldrich was used as such. Zeolite-Y and ZSM-5 obtained from Sud Chemie, India are washed with $1 \mathrm{M} \mathrm{NaCl}$ for about 2 hours and calcined at $530^{\circ} \mathrm{C}$ for $12 \mathrm{~h}$. Cetyltrimethylammonium bromide (CTAB) and tetraethylorthosilicate (TEOS) were obtained from Lancaster. All other chemicals were purchased from Qualigens and Merck fine chemicals. Standard methods are followed for the purification of the common organic solvents. All other chemicals of analytical grade are used as received unless otherwise stated. Water used in this investigation was triply distilled over alkaline potassium permanganate

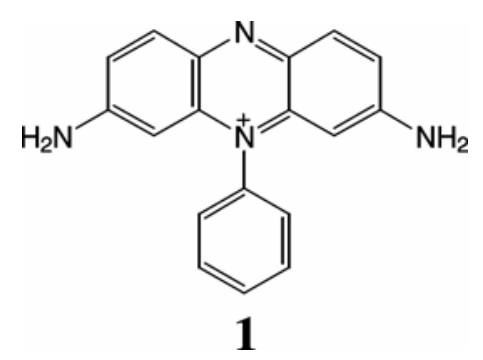

in an all glass apparatus and was used for all the experiments. 


\subsection{Synthesis of MCM-41 and modified MCM-41}

MCM-41 was synthesised by following the procedure already reported in the literature. ${ }^{27} 2.4 \mathrm{~g}$ of $n$-cetyltrimethylammoniumbromide, CTAB was dissolved in $120 \mathrm{~g}$ of deionised water and stirred until the solution was homogeneous and clear. Ammonium hydroxide $(8 \mathrm{ml})$ was added to this mixture and stirred for $5 \mathrm{~min}$ after which $10 \mathrm{~mL}$ of tetraethylorthosilicate was added to give a molar composition of the gel (1 M TEOS: $1.64 \mathrm{M} \mathrm{NH}_{4} \mathrm{OH}$ : $0.15 \mathrm{M}$ CTAB: $126 \mathrm{M} \mathrm{H}_{2} \mathrm{O}$ ). The reaction mixture was stirred overnight, after which the solution was filtered and washed several times consecutively with deionised water and ethanol; calcination was performed at $823 \mathrm{~K}$ for $5 \mathrm{~h}$.

The modification of the interior structure of MCM41 was carried out through the use of a silylation reagent 3-aminopropyltriethoxysilane, APTES. The silane modified MCM-41 (SMCM-41) was prepared according to a procedure detailed. ${ }^{28}$ About $0.6 \mathrm{~g}$ of the calcined MCM-41 was mixed in a chloroform solution of 3-APTES $(50 \mathrm{~mL}, 0 \cdot 15 \mathrm{M})$ and stirred overnight at room temperature. The precipitate was filtered and washed with chloroform and dichloromethane. Aluminosilicate mesoporous materials were prepared by post synthesis method by using aqueous aluminium chlorohydrate containing aluminium polycations as reported earlier. ${ }^{29}$ The obtained material was characterized by SAXRD and surface area analysis and found to be showing identical characteristics as reported earlier. ${ }^{30}$

\subsection{Encapsulation of phenosafranine in mesoporous hosts by impregnation method}

Phenosafranine dye encapsulated in MCM-41, AlMCM-41 or SMCM-41 composites were prepared by stirring the aqueous solution of the dye and the mesoporous materials for six to eight hours at room temperature which results in the loading of phenosafranine in mesoporous hosts. The material was filtered, washed thoroughly until the filtrate shows no absorbance at $520 \mathrm{~nm}$. The amount of the dye loaded in mesoporous hosts was adjusted by taking different amounts of dye in aqueous solution. Phenosafranine coated on silica was prepared by stirring an aqueous solution of the dye with the calculated amount of silica for about $6 \mathrm{~h}$. The resulting pink coloured solid was filtered and washed with excess amount of water until the filtrate showed no absorbance at $520 \mathrm{~nm}$.
2.4 Adsorption of phenosafranine in microporous hosts byion exchange method

The aqueous solution of the dye $\mathrm{PS}^{+}$and the zeolite were stirred for three to four hours at room temperature, which resulted in the $\mathrm{PS}^{+}$ion exchanged with the cations present in the zeolite samples. The concentration of the dye in zeolite was maintained at $3 \mu \mathrm{M}$ per gram of the zeolite-Y and $2.3 \mu \mathrm{M}$ per gram of ZSM-5.

\subsection{Instrumentation}

X-ray powder diffraction data of the MCM-41 material was collected on a Pananalytical X'pert Pro $\mathrm{X}$-ray diffractometer. XRD patterns were obtained between 0.5 and $10.2 \theta$. Adsorption and desorption isotherms were measured at $77 \mathrm{~K}$ on a Micromeritics ASAP 2010 instrument using nitrogen gas. The samples were outgassed at $423 \mathrm{~K}$ and $1 \mathrm{mPa}$ for $14 \mathrm{~h}$ before adsorption measurements. The specific surface area of these mesoporous materials was determined by the standard BET method. The X-ray photoelectron spectra (XPS) of the samples were recorded on a Kratos-Axis 165 XPS spectrometer with $\mathrm{MgK}$ radiation $(1253.6 \mathrm{eV})$ at $75 \mathrm{~W}$. The $\mathrm{C} 1 \mathrm{~s}$ line at $284.6 \mathrm{eV}$ was used as an internal standard for the correction of binding energies. UV-visible diffuse reflectance spectral studies of the samples were recorded using Agilent 8453 diode array spectrophotometer equipped with labsphere RSA-HP-8453 reflectance accessory.

\section{Results and discussion}

\subsection{XPS analysis of phenosafranine adsorbed on micro and mesoporous materials}

Comparison of the binding energy (BE) of free and surface adsorbed dye is an ideal method to understand the nature of host-guest interaction. ${ }^{29,31}$ The present XPS studies are concerned with the nitrogen atoms of the dye since $\mathrm{C} 1 \mathrm{~s}$ BE remains significantly unaltered for the dye adsorbed on the surface of hosts. The core level X-ray photoelectron spectra of crystalline phenosafranine (figure 1) shows broad peak for ls level of carbon and it can be resolved into three peaks with binding energies (BE) of $284.8,286.2$ and $288.2 \mathrm{eV}$. The three peaks are due to the difference in the electronegativity of the 
bonded neighbouring atom. The binding energy of $284.8 \mathrm{eV}$ for $1 \mathrm{~s}$ carbon, which has the maximum peak area among the observed Cls peaks is attributed to the carbon bonded to another carbon atom. The next higher energy peak observed at 286.2 corresponds to the carbon bonded to nitrogen atom. The more electronegative nitrogen atom attracts the bonding electrons towards its nucleus, which results in the increase in the charge of the nucleus inturn enhancing the binding energy of the $\mathrm{Cls}$ electron. The peak at $288.2 \mathrm{eV}$ is assigned to the carbon of $\mathrm{C}-\mathrm{N}^{+}$; presence of positive charge on the nitrogen atom attracts the bonding electron, which in turn increases the binding energy of the $\mathrm{Cls}$ electron to which it is bound.

The core level nitrogen N1s peak also appears as a broad peak with high full width at half maxima (FWHM) which could be resolved into three peaks. In $\mathrm{PS}^{+}$, four nitrogen atoms are present and among these two of them are in identical environment. The lower binding energy for nitrogen $(398.5 \mathrm{eV})$ is assigned to the nitrogen atom of two primary amino groups. The area under the curve, which is about $58 \%$ of the observed broad peak confirms this assignment. The next high-energy peak due to the nitrogen atom of the azine ring shows the binding energy of $1.2 \mathrm{eV}$ higher than that of the primary
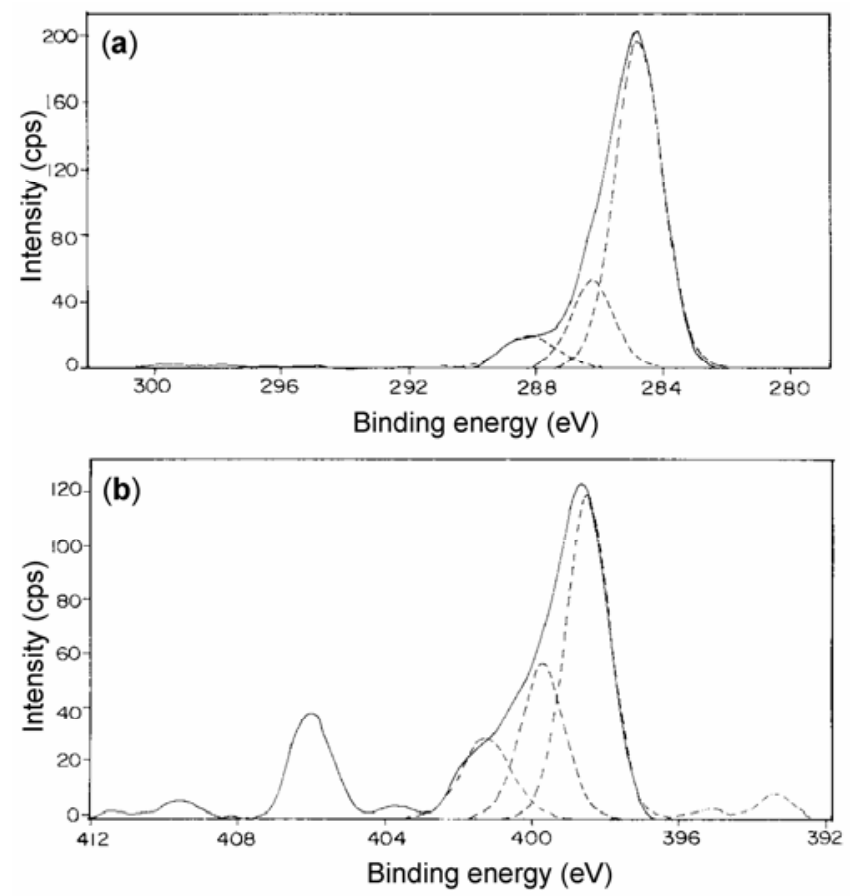

Figure 1. XPS core level spectra of crystalline phenosafranine dye (a) C1s and (b) N1s. amino nitrogen. This increase in binding energy is due to the aromaticity of the azine ring. Aromaticity is known to have large impact on the binding energies, which is due to the delocalisation of electrons in aromatic molecules. ${ }^{31}$ The third high binding energy of Nls is due to the presence of positive charge on the nitrogen atom. There are two nitrogen atoms present in the azine ring and both of them are responsible for the aromaticity of the central ring of phenazine moiety. In one of the ring nitrogen phenyl group is attached. Generally, the positive charge is stabilised at the particular atom, which is bound to more electron donating groups. Hence the nitrogen atom with positive charge is attributed to the nitrogen attached to the phenyl ring. The phenyl ring is out of the azine plane which increases the electron density around the nitrogen atom, stabilizing the positive charge on that nitrogen. The small discrepancies in the integrated area under the curve are attributed to the delocalisation of the positive charge in the phenazine ring. The most favourable form among the possible canonical forms of $\mathrm{PS}^{+}$is positive charge on the nitrogen bonded with phenyl group as indicated below.

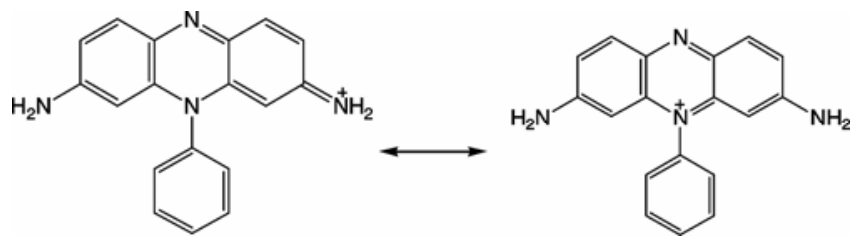

The core level X-ray photoelectron spectra of phenosafranine encapsulated in MCM-41 (figure 2) shows those $\mathrm{C} 1 \mathrm{~s}$ binding energies to be $284 \cdot 6,286 \cdot 4$ and $288.3 \mathrm{eV}$. The peaks are assigned to the $\mathrm{C}-\mathrm{C}$, $\mathrm{C}-\mathrm{N}$ or $\mathrm{C}=\mathrm{N}$ and $\mathrm{C}-\mathrm{N}^{+}$similar to that of the free dye. On careful examination of this data, it is inferred that the binding energies of the carbon atoms do not change significantly. The electronic configuration of the carbon atom as suggested by the XPS data is not that much perturbed on the MCM-41 surface which shows that the interaction between the MCM-41 surface and the dye molecule is primarily through the nitrogen atoms of $\mathrm{PS}^{+}$.

The interaction between the dye and the MCM-41 surface is obtained from the XPS of nitrogen 1s level. It is interesting to note that the observed broad peak is best fitted with two-peak fit rather than three as in the case of free dye and the dye adsorbed on the outer surface of silica. This indicates that the two primary amino groups of phenosafranine are in 
identical environments and the two ring nitrogens are also in identical environments. The BE of N1s level corresponds to 398.3 and $400 \cdot 1 \mathrm{eV}$. The lower binding energy peak at $398.3 \mathrm{eV}$ is due to the primary amino nitrogen atoms present in the phenosafranine dye molecule. The presence of hydrogen bonding interaction between the nitrogen atoms of phenosafranine with the MCM-41 surface forces the primary amino groups out of the azine plane, which enhances the electron density around these nitrogen atoms which results in the decrease in binding energy of N1s. The high BE peak is attributed to the nitrogen interacting with the surface hydroxyl group through hydrogen bonding which corresponds to $\sim 51 \%$ of the total intensity. The increase in binding energy of about $1 \mathrm{eV}$ for ring nitrogen, is presumably due to the hydrogen bonding with the hydrogen of surface hydroxyl group of MCM-41. The nitrogen atom is involved in a hydrogen bonding interaction and its electron density is lower since the electrons are partially shared with more electronegative atoms. In the present case, electron density present at nitrogen is shared with the hydrogen atoms which are electron deficient due to the binding with oxygen; as a consequence the N1s binding energy increases.

Comparison of the binding energy (BE) of free and surface adsorbed dye is useful to understand the
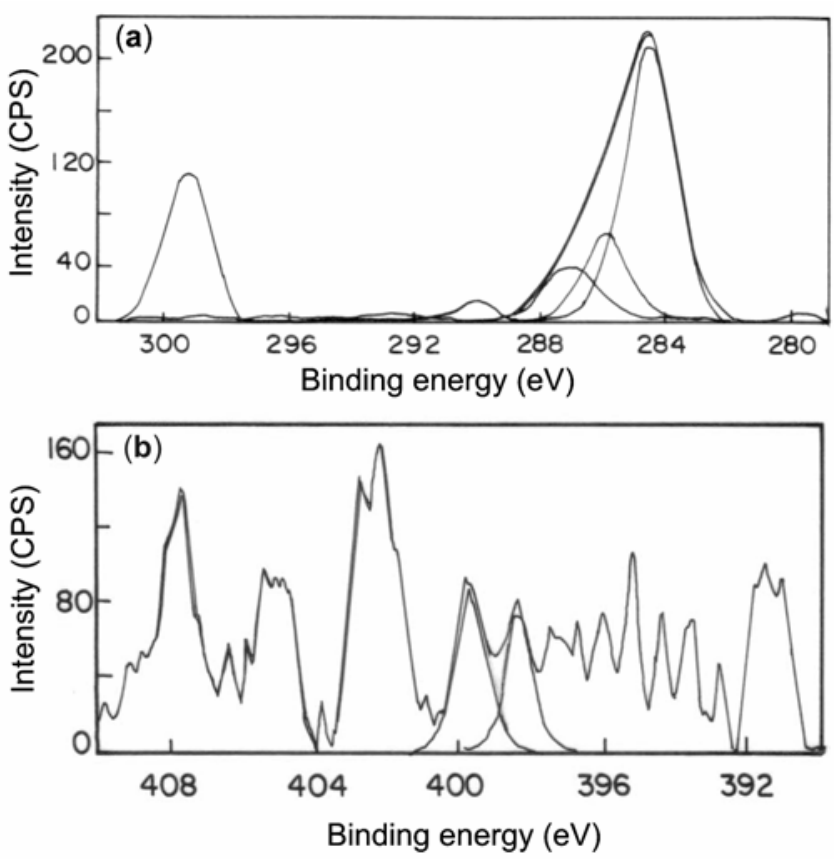

Figure 2. (a) C1s; (b) N1s XPS spectrum of phenosafranine in MCM-41. nature of host-guest interaction. The present XPS studies are concerned with the nitrogen atoms of the dye since $\mathrm{C} 1 \mathrm{~s} \mathrm{BE}$ remains significantly unaltered for the dye adsorbed on the surface of hosts. Out of four nitrogen atoms present in $\mathrm{PS}^{+}$two of them are in identical environment in pure crystalline form, i.e. primary amino groups (BE $398.3 \mathrm{eV})$. The other two nitrogen atoms are located in the central aromatic ring $(\mathrm{BE} 399 \cdot 5,400 \cdot 3 \mathrm{eV})$. Aromaticity is known to have larger impact on $\mathrm{BE}$ due to the delocalisation of electrons in the aromatic ring which reduces electron density around the nitrogen atom leading to higher binding energy. ${ }^{32}$ Among the two aromatic nitrogen atoms, $400 \cdot 3 \mathrm{eV}$ peak is assigned to the aromatic nitrogen bonded to the phenyl ring, where the positive charge is more localised due to the electron donating nature of the phenyl ring, which stabilises the positive charge more favourably than the other nitrogen atoms.

The N1s BE values of $\mathrm{PS}^{+}$adsorbed on the silica surface are presented in table 1 . The BE values, show that $\mathrm{N} 1 \mathrm{~s}$ BE values are significantly altered indicating that the interaction between the dye and the host surface is through nitrogen atoms. The BE of primary amino group is found to increase by about $2.0 \mathrm{eV}$ at the silica surface due to H-bonding between hydrogen of amino group and the oxygen of surface hydroxyl groups. The observed decrease in the $\mathrm{N}$ 1s BE values of ring nitrogen in $\mathrm{PS}^{+}$is due to the fact that the adsorption at the surface causes deformation of the aromatic ring which in turn leads to a decrease in the delocalisation of electron in the ring. 32,33

The observed and deconvoluted core level N1s XPS of $\mathrm{PS}^{+}$in zeolite is shown in figure 2. The BE values of the deconvoluted peaks presented indicate that the mode of interaction between the dye and the zeolite surface is through $\mathrm{H}$-bonding as in the case of silica. Even though the primary amino groups of $\mathrm{PS}^{+}$are in identical environments, the difference in $\mathrm{BE}$ between them is due to the loss of symmetry in the molecule due to adsorption of the dye at the zeolite surface. In principle, for a completely planar molecule there should be an equivalent electronic distribution around the two nitrogen atoms and hence a sharp N1s peak is predicted to be observed. For the slightly distorted conformer since the two nitrogen atoms are not equivalent a break in conjugation results in the broadening of XPS band. ${ }^{34}$ Accordingly, in the present case the loss of symmetry in the molecule is due to one of the nitrogen 
Table 1. X-ray photoelectron spectroscopic data of phenosafranine encapsulated in micro and mesoporous materials.

\begin{tabular}{lcc}
\hline Sample & Carbon $(1 \mathrm{~s}) \mathrm{BE}$ value $(\mathrm{eV})$ & Nitrogen $(1 \mathrm{~s}) \mathrm{BE}$ value $(\mathrm{eV})$ \\
\hline Phenosafranine solid dye & $284 \cdot 8\left(74 \cdot 2 * ; 1 \cdot 74^{\#}\right)$ & $398 \cdot 5(57 \cdot 5 ; 1 \cdot 34)$ \\
& $286 \cdot 2(17 \cdot 9 ; 1 \cdot 54)$ & $399 \cdot 2(26 \cdot 7 ; 1 \cdot 32)$ \\
Phenosafranine adsorbed on silica & $288 \cdot 2(7 \cdot 9 ; 1 \cdot 92)$ & $401 \cdot 2(15 \cdot 8 ; 1 \cdot 51)$ \\
& $284 \cdot 6(51 \cdot 8 ; 1 \cdot 74)$ & $398 \cdot 3(24 \cdot 2 ; 1 \cdot 03)$ \\
& $286 \cdot 3(31 \cdot 8 ; 1 \cdot 64)$ & $399 \cdot 5(26 \cdot 9 ; 0 \cdot 67)$ \\
Phenosafranine encapsulated in MCM-41 & $288 \cdot 4(16 \cdot 4 ; 3 \cdot 02)$ & $400 \cdot 3(48 \cdot 9 ; 1 \cdot 20)$ \\
& $286 \cdot 4(33 \cdot 3 ; 1 \cdot 69)$ & $398 \cdot 3(49 \cdot 4 ; 1 \cdot 40)$ \\
Phenosafranine adsorbed on ZSM-5 & $288 \cdot 3(12 \cdot 4 ; 1 \cdot 90)$ & $400 \cdot 1(50 \cdot 6 ; 1 \cdot 49)$ \\
& $284 \cdot 5(67.8 ; 1 \cdot 38)$ & $399 \cdot 2(41.5 ; 1.50)$ \\
Phenosafranine adsorbed on zeolite-Y & $285.5(19 \cdot 6 ; 1 \cdot 27)$ & $400 \cdot 8(58 \cdot 5 ; 2 \cdot 27)$ \\
\hline
\end{tabular}

*Relative intensity; ${ }^{\#}$ Full width at half maximum (FWHM)

atoms bind strongly to the substrate and the other remains free above the surface as depicted in scheme. Alternately, the loss of symmetry could also result from the heterogeneous nature of the zeolite surface. The hydrogen bonding interaction between the hydrogen of amino group with the silanol and aluminol groups reduces the symmetry of the dye molecule adsorbed at the zeolite surface. These two factors are presumably responsible for the observed difference in $\mathrm{BE}$ between the two nitrogen atom of the primary amino groups. However, the higher BE value of primary amino nitrogen atoms suggests that hydrogen bonding with the surface hydroxyl groups exists with the primary amine hydrogen atoms and hence it is concluded that the surface heterogeneity is solely responsible for the observed behaviour. The observed small variations of nitrogen $1 \mathrm{~s} \mathrm{BE}$ in titanium dioxide anchored on the external surface of the zeolite (TYS) samples are due to the change in acidity of the zeolite surface by titanol groups, which affects the hydrogen bonding energy.

In contrast to zeolite-Y and silica, the nitrogen $1 \mathrm{~s}$ peak of $\mathrm{PS}^{+}$in the ZSM-5 surface could be deconvoluted into two peaks. The higher BE value of nitrogen is assigned to the nitrogen of primary amino group which is involved in the hydrogen bonding with the surface hydroxyl groups. Contrary to the $\mathrm{PS}^{+}$-zeolite-Y, PS ${ }^{+}$-ZSM-5 shows one BE value for primary amino nitrogen atoms even though the latter surface is also heterogeneous in nature. The observed result is attributed to the broad peak for N1s level. It is worth noting that the BE of nitrogen $1 \mathrm{~s}$ level of $\mathrm{PS}^{+}$at ZSM-5 surface is significantly less than that observed in zeolite-Y. The smaller BE value of nitrogen $1 \mathrm{~s}$ level reveals that the hydrogen bonding is weak in the case of ZSM-5 as compared to that in zeolite-Y. When the ZSM-5 surface is modified with titanium dioxide nanoparticles the nitrogen 1s level of $\mathrm{PS}^{+}$could be deconvoluted into three Gaussian peaks. The BE values for nitrogen of the primary amino groups is attributed to the loss of symmetry in $\mathrm{PS}^{+}$molecule at the ZSM-5 surface as well similar to that observed in zeolite-Y.

Phenosafranine dye in aqueous solution shows absorption band with maximum at $520 \mathrm{~nm}$ in the visible region with the absorption maximum not affected by the nature of the solvent used. The dye adsorbed on chromatographic grade silica also absorbs at $520 \mathrm{~nm}$. The diffuse reflectance spectrum of phenosafranine $\left(\mathrm{PS}^{+}\right)$encapsulated in MCM-41 surface shows the absorption band with maximum at $524 \mathrm{~nm}$ for hydrated samples of MCM-41. The absorption band is red shifted as compared to that observed in homogeneous solution. The red shift observed is attributed to the interaction of the dye with the MCM-41 matrix. Dehydration of the dye loaded zeolite samples at $125^{\circ} \mathrm{C}$ in vacuum does not alter the absorption maxima. However, a small fraction of the dye is decomposed as revealed from decrease in absorbance. Generally, dehydration leads to a change in surface polarity of the MCM-41 due to the removal of water molecules present. The absorption maxima observed 
at same wavelength for phenosafranine adsorbed on different solid surfaces, with its hydrated and dehydrated form reveals the insensitivity of the dye towards surface polarity of the mesoporous materials. The observed behaviour is akin to that observed for homogeneous solution of dye in different solvents.

The photophysical properties of fluorescent probes are influenced by the host environment which explains the host-guest interaction between the solid surface of the host and the dye molecules. The photophysical properties of $\mathrm{PS}^{+}$in ZSM-5, silica and the dye loaded zeolite samples after dehydration indicate that the absorption and fluorescence spectral properties of hydrated form of $\mathrm{PS}^{+}$in ZSM-5 and titanium dioxide loaded ZSM-5 are similar to that of zeolite-Y published earlier. ${ }^{8}$

Dehydration of the samples does not seem to alter the absorption maxima of the dye in zeolite- $Y$ and ZSM-5 hosts. However, a small decrease in absorbance reveals that a fraction of the dye may have been decomposed. Generally, dehydration leads to some changes in the surface polarity of the zeolite due to the removal of the water molecules present at the surface. ${ }^{28}$ The absorption maxima observed at $520 \mathrm{~nm}$ for $\mathrm{PS}^{+}$adsorbed on different solid surfaces, with its hydrated and dehydrated form shows that the surface polarity of the zeolites does not affect the
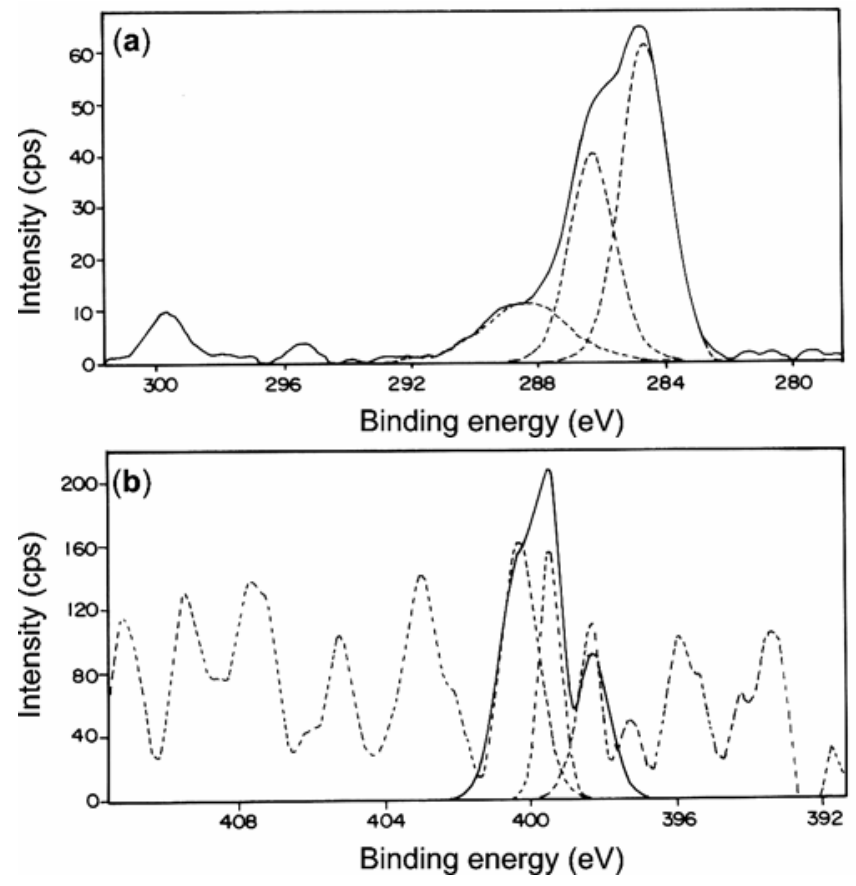

Figure 3. XPS core level spectra of phenosafranine adsorbed on silica (a) C1s and (b) N1s. dyes significantly in the ground state. The observed behaviour is similar to that observed for homogeneous solution of dye in different solvents. Dehydrated form of the dye in titanium dioxide loaded zeolite samples shows absorption maximum at $530 \mathrm{~nm}$ which is a shift of $10 \mathrm{~nm}$ towards red as compared to the hydrated forms of TSY and TSZ samples; in the case of TEY, TEZ samples the observed red shift is $10 \mathrm{~nm}$. The shift in absorption maximum towards longer wavelength is obviously due to the protonation of the dye molecules. Significant quantity of the adsorbed dyes on zeolite surface also gets decomposed during the dehydration process as revealed from the decrease in absorbance.

\section{Conclusion}

The XPS studies of the dye incorporated host show that the binding energy for carbon $1 \mathrm{~S}$ remains significantly unaltered. The dye molecule interacts with the host surface through hydrogen bonding between
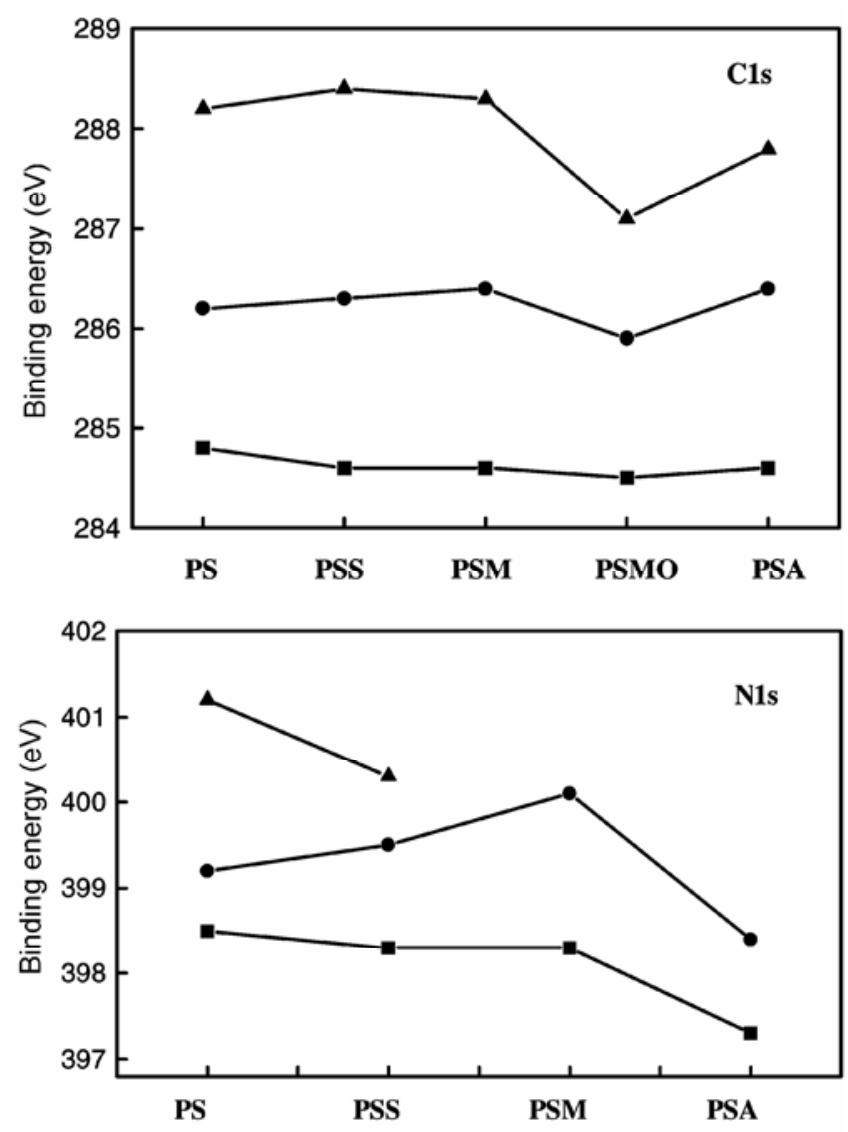

Figure 4. Binding energy plot of phenosafranine C1s and N1s in various mesoporous hosts. 
the hydrogens of the primary amino group and oxygen of surface hydroxyl groups as revealed from the XPS studies. However, the strength of the hydrogen bonding is highly sensitive to the nature of the surface. The ground state properties of phenosafranine encapsulated mesoporous materials are not sensitive to the nature of the surface.

\section{Acknowledgements}

The authors acknowledge the financial support received from the Department of Science and Technology (DST), Government of India through the Raja Ramanna Fellowship to P N. The centre is supported by DST, Intensification of Research in High Priority Areas (IHRPA) programme.

\section{References}

1. Thomas J M 1999 Angew. Chem. Int. Ed. 383588

2. Sayari A 1996 Chem. Mater. 81840

3. Hagfeldt A and Gratzel M 1995 Chem. Rev. 9549

4. Calzaferri G, Pauchard M, Maas H, Huber S, Khatyr A and Schaafsma T 2002 J. Mater. Chem. 121

5. Calzaferri G, Maas H, Pauchard M, Pfenniger M, Megelski S and Devaux A 2002 Adv. Photochem. 27 1

6. Hashimoto S $2003 \mathrm{~J}$. Photochem. Photobiol. C: Photochem. Rev. 419

7. Ananthanarayanan K, Selvaraju $\mathrm{C}$ and Natarajan $\mathrm{P}$ 2007 Micropor. Mesopor. Mater. 99319

8. Easwaramoorthi S and Natarajan P 2005 Micropor. Mesopor. Mater. 86186

9. Scott B J, Wirnsberger G and Stucky G D 2001 Chem. Mater. 133140

10. Ogawa M 2002 J. Photochem. Photobiol. C. Photochem. Rev. 3129

11. Hoppe R, Ortlam A, Rathousky J, Schulz-Ekloff G and Zukal A 1997 Micropor. Mater. 8267

12. Yamashita $H$, Nishimura M, Koyano K, Tatsumi $T$ and Anpo M 1998 Stud. Surf. Sci. Catal. 117551

13. Gu G, Ong P P and Li O T 1999 J. Phys. D: Appl. Phys. 322287
14. Ogawa M, Nakamura T, Mori J and Kuroda K 2000 J. Phys. Chem. B104 8554

15. Xu O, Li L, Liu X and Xu R 2002 Chem. Mater. 14 549

16. Xei W, Aydin M, Zakia S and Akins D L $2004 \mathrm{~J}$. Phys. Chem. B108 5588

17. Guo H, Zhang X, Aydin M, Xu W, Zhu H R and Akins D L 2004 J. Molec. Struct. 689153

18. Li D, Zhao W, Sun X, Zhang J, Anpo M and Zhao 2006 J. Dyes Pigments 6833

19. Wang L, Liu Y, Chen F, Zhang J and Anpo M 2007 J. Phys. Chem. C111 5541

20. Alvaro M, Ferrer B, Garcia H, Palomares E J, Balzani V, Credi A, Venturi M, Stoddart J F and Wenger S 2003 J. Phys. Chem. B107 14319

21. Gao Y, Konovalova T A, Lawrence J N, Smitha M A, Nunley J, Schad R and Kispert L D 2003 J. Phys. Chem. B107 2459

22. Broglia MF, Bertolotti S G, Previtali C M and Montejano H A $2006 \mathrm{~J}$. Photochem. Photobiol. A: Chem. 180143

23. Ramaraj R and Natarajan P 1989 J. Chem. Soc. Faraday Trans. $\mathbf{8 5} 813$

24. Gopidas K R, Weir D and Kamat P V 1988 Chem. Phys. Lett. 149491

25. Chaudhuri R, Sengupta P K and Rohatgi Mukherjee K K 1997 J. Photochem. Photobiol. A: Chem. 108261

26. Natarajan P and Raja C 2005 Eur. Polym. J. 412496

27. Kumar D, Schumacher K, Von Hohenesche C D F, Grun M and Unger K K 2001 Colloids and Surfaces A: Physiochemical and Engineering Aspects 109187

28. Uppili S, Thomas K J, Crompton E M and Ramamurthy V 2000 Langmuir 6265

29. Vieira Ferreira L F, Cabral P V, Almeda P, Oliveira A S, Reis M J and Botelho do Rego A M 1998 Macromolecules 313936

30. Ananthanarayanan K and Natarajan P 2009 Micropor. Mesopor. Mater. 124179

31. Vieira Ferreira, LF, Lemos M J, Reis M J and Botelho do Rego A M 2000 Langmuir 165673

32. Ferreira L F V, Lemos M J, Reis M J and do Rego A M B 2000 Langmuir 125673

33. Kerber S J, Bruckner J J, Wozniak K, Seal S, Hardcastle S and Barr T L 1996 J. Vac. Sci. Technol. A14 1314

34. Do Rege, A M B, Pereira L P, Reis M J, Oliveria A S and Ferreira L F V 1997 Langmuir 136787 